\title{
Research of Networked Control System Based on Fuzzy Adaptive PID Controller
}

\author{
Daogang Peng, Hao Zhang, Hui Li, and Fei Xia
}

\begin{abstract}
Networked control system has recently been a hotspot in the research fields of control theory and control engineering application, which has the advantages of resource sharing, fewer cables, easy to extend and maintain, etc. As the controlled object includes complicated control network and physical object, the controller of networked control system can adopt fuzzy control method. Taking the typical second-order inertia plant in the process of industrial production for example and using the TrueTime toolbox in Matlab, the networked control system based on fuzzy adaptive PID controller had been designed. Through the simulation studies of changing parameters and transmission rates, increasing network delay, proved that the control method can get better control effects.
\end{abstract}

Index Terms-Networked control system, fuzzy adaptive pid controller, fuzzy control, truetime toolbox.

\section{INTRODUCTION}

Networked control system has been a hotspot in the research fields of control theory and control engineering application in the late 1990s. Each node in the networked control system is connected to network directly, so it has the advantages of resource sharing, fewer cables, easy to extend and maintain, etc [1]. As the controlled object includes complicated control network and physical object, meanwhile, fuzzy control is convenient when the effect of traditional control method is not quite good or the object model is unknown, the controller of networked control system can adopt fuzzy control method [2]. The algorithms of fuzzy adaptive PID controller is combined with fuzzy control rules and PID controller, whose inputs are error and error change, and the output is three changing values of PID parameters. This kind of algorithm, changes PID parameters according to fuzzy reasoning made by fuzzy control rules, can greatly improve the control performance.

The TrueTime toolbox is a simulation platform based on Matlab/Simulink for networked control system. The corresponding real-time networked control system can be built by connecting the modules in TrueTime and the common modules in Simulink, which can provide basis for the simulation study of delay compensation algorithm of the networked control system and scheduling algorithms of different resources [3], [4]. Taking the typical second-order

Manuscript received September 9, 2013; revised November 14, 2013 This work was supported in part by the State Key Program of National Natural Science Foundation of China under Grant 61034004 and Shanghai Science and Technology Commission Key Program under Grant 13111104300 .

The authors are with the Shanghai University of Electric Power, Shanghai 200090, China (e-mail: jypdg@163.com, hzhangk@163.com, elmerlee@163.com, xiafeiblue@163.com). inertia plant in the process of industrial production for example, and using the TrueTime toolbox, the networked control system based on fuzzy adaptive PID controller has been designed in this paper. Through the simulation study of increasing time delay, changing parameters and transmission rate, we can see that the control method designed in this paper gets a better control effect with a better ability of robustness, adaptability and anti-interference.

\section{FUZZY ADAPTIVE PID NETWORKED CONTROL SYSTEM}

\section{A. System Designation}

The essence of the networked control system is that through the network, the messages of reference input, object output and control input exchange data between different components of networked control system, such as sensors, controllers and actuators and so on .These devices belong to the network nodes in the networked control system. Every node can execute several different tasks according to its own hardware configuration. Such as monitor computer to receive data of the control layer through net, implement optimized algorithm and download the optimized parameters to the related nodes of control layer in the field through the net. Each node in the networked control system is connected to the network directly, so it has the advantages of resource sharing, fewer cables, easy to extend and maintain, etc.

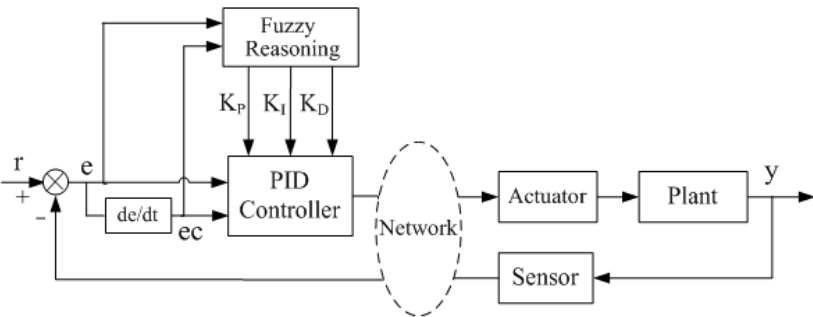

Fig. 1. Structure of fuzzy adaptive PID networked control system.

The structure of networked control system based on fuzzy adaptive PID controller is shown in Fig. 1, where sensors are used to sample signals periodically from controlled objects on time driving and pass them to the fuzzy adaptive PID controller through network communication channels, then controller calculates the control signals via fuzzy adaptive PID algorithm and pass them to the actuators by network communication channels, finally the actuators control the controlled object in accordance with the results of fuzzy adaptive PID controller. The controllers and actuators use either time-driven mode or event-driven mode. The controller and actuator calculate and execute the latest date packet information in a steady time interval when they are both time-driven mode. On the other side, when they both use 
event-driven mode, the controller calculates the new control variable when the controller received a date packet and the actuator doesn't work until it receives the date packet of control variable [5].

The simulation study of fuzzy adaptive PID networked control system can be finished by using TrueTime toolbox based on Matlab. The TrueTime toolbox is a simulation platform based on Matlab/Simulink for networked control system, which is developed by Martin Ohlin, Dan Henriksson and Anton Cervin, etc of Sweden Lund University. There are six main modules in the TrueTime toolbox, namely TrueTime Kernel module, TrueTime Network module, TrueTime Wireless Network Module, ttSendMsg module, ttGetMsg module and TrueTime Battery module. The corresponding real-time networked control system can be built by connecting the modules in TrueTime and the common modules in Simulink. The dynamic process of networked control system, control task execution and co-simulation environment of network interaction can be built by the simulation module of simulink in the MATLAB. The simulation study of delay compensation algorithm of the networked control system and scheduling algorithms of different resources can be carried out. The TrueTime Kernel module in the TrueTime box can be used to simulate nodes of networked control system, such as sensors, controllers and actuators. It has flexible real-time kernel, A/D and D/A converters, network sending and receiving interfaces. It also supports four kinds of task scheduling algorithms, such as fixed priority (prioFP), rate monotonic (prioRM), deadline monotonic (prioDM) and earliest deadline first (prioEDF). The TrueTime Network module uses event-driven mode, when the messages are read in or sent out, the module executes the task. The messages read in or sent out should contain the information about the sending and receiving computer nodes, user defined control signals or measured signal data, the total length of the message, real-time characteristic parameters such as priority, deadline and so on.

\section{B. Algorithm of Fuzzy Adaptive PID Controller}

The basic ideal of fuzzy control is to use a machine on the fuzzy model for system control instead of a person, it is a computer numerical control method based on fuzzy set theory, fuzzy language variables and fuzzy reasoning. Fuzzy control belongs to non-linear control and intelligent control, it is especially useful when the mathematical models of controlled object is unknown or quite complex. The design theory and control algorithm of fuzzy control has been a hot spot in the field of control theory, and the key of designing a fuzzy controller is to find appropriate fuzzy control rules for corresponding object. Generally speaking, the fuzzy control rules are established based on knowledge and experience of experts or experienced operators, according which the designers design corresponding fuzzy controllers to complete control tasks.

As fuzzy control is convenient when the effect of traditional control method is not quite good or the object model is unknown, meanwhile, the control object includes complicated control network and physical object, the controller of networked control system can adopt fuzzy control method.
The networked control system in this paper is carried out by fuzzy adaptive PID control algorithm, whose inputs are error and error rate, and the output is three changing values of PID parameters. This kind of algorithm realize self-adjusting by changing PID parameters on-line according to fuzzy reasoning made by fuzzy control rules, then the modified PID parameters are taken to conventional PID so as to improve control performance of control system. The fuzzy controller used here is based on Max-Min-Center of Gravity method put forward by Mamdani. The process of fuzzy adaptive PID control is firstly to find the relations among $e, e c$ and PID parameters, secondly detect $e$ and $e c$ continuously during the operation and then modify PID parameters on-line according to fuzzy control theory to meet requirements of $e$ and $e c$ in different stages.

The principles of fuzzy adaptive PID control algorithm are as followes:

1) When the controlled variable is close to given value, the integral action which has the same sign as ec can avoid overshoot and oscillation. On the opposite side, when the controlled variable is far from given value, the proportional action which has the opposite sign to $e$ can decrease overshoot and avoid oscillation.

2) At prophase, increase proportional action appropriately and define integral value a smaller value or zero so as to avoid overshoot and improve response speed; At metaphase, the proportional and integral actions are well situated to have a good effect of stability and control precision. At anaphase, decrease proportional action and increase integral action to eliminate error and reduce overshoot.

3) The differentiation, the function of which is to reduce changes of controlled variables, shorten regulate time and eliminate error, is a supplement of proportional-integral.

With fuzzy control rule tables of $K_{P}, K_{I}$ and $K_{D}$, the fuzzy control models of every parameters and valuated tables of different membership degrees, through the designed fuzzy matrix tables of PID parameters, and then insert the modified parameters in next formulas:

$$
\begin{aligned}
& K_{P}^{\prime}=K_{P}+\{e, e c\}_{P} \\
& K_{I}^{\prime}=K_{I}+\{e, e c\}_{I} \\
& K_{D}^{\prime}=K_{D}+\{e, e c\}_{D}
\end{aligned}
$$

During the on-line operation, the results of fuzzy logic rules are handled and calculated by control system and then the PID parameters are corrected on line, whose working flow chart is shown in Fig. 2.

The designation of fuzzy adaptive PID controller is as follows:

\section{1) Input variables}

The input variables of fuzzy controller are error $e$ and error change $e c$, whose fuzzy domain is $[-3,3]$ and fuzzy set of linguistic variables is $\{\mathrm{NB}, \mathrm{NM}, \mathrm{NS}, \mathrm{ZE}, \mathrm{PS}, \mathrm{PM}, \mathrm{PB}\}$. The function of membership degree here adopts piecewise linear function.

The error and error change are in the range of [-emax, emax] and [-ecmax, ecmax] during practical operation, so [-emax, emax] and [-ecmax, ecmax] are named basic universe of 
$e$ and $e c$. Interval transforms are needed by quantization factors $k e$ and $k d e$, which are defined as bellowing: $k e=3 /$ emax, $k d e=3 /$ demax.

\section{2) Output variables}

The output variables of fuzzy controller are $\mathrm{kup}=f 1(\mathrm{e}, \mathrm{de})$, kui= $f 2(e, d e)$, and kud=f3 $(e, d e)$, whose fuzzy domain is $[-3,3]$ and fuzzy set of linguistic variables is \{NB,NM,NS,ZE,PS,PM,PB $\}$. The basic universes of kup, kui and kud are [-kupmax, kupmax], [-kuimax, kuimax] and [-kudmax, kudmax], and define scale factors $\mathrm{kbp}=\mathrm{kupmax} / 3$, $\mathrm{kbi}=\mathrm{kuimax} / 3$ and $\mathrm{kbd}=\mathrm{kudmax} / 3$.

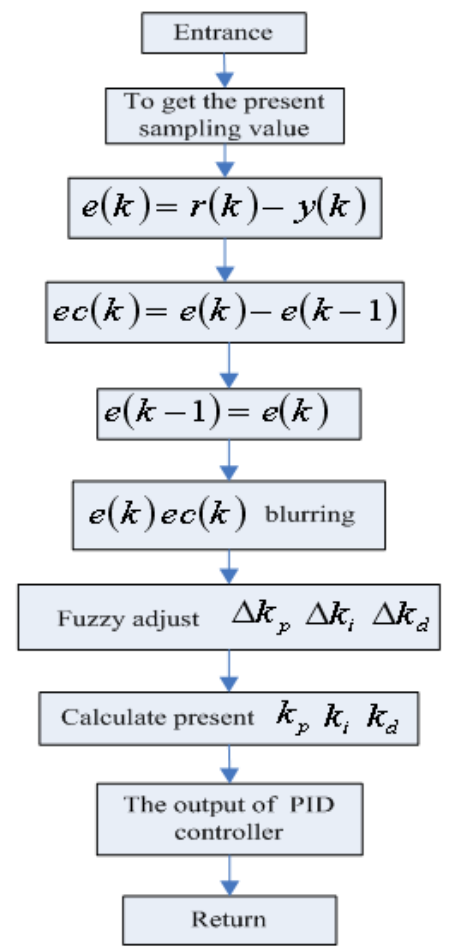

Fig. 2. The working flow chart of fuzzy adaptive PID controller.

TABLE I: FUZZY CONTROL RULES TABLE

\begin{tabular}{|c|c|c|c|c|c|c|c|c|}
\hline \multirow{2}{*}{\multicolumn{2}{|c|}{$\begin{array}{l}\Delta K_{P} \\
\Delta K_{I} \\
\Delta K_{D}\end{array}$}} & \multicolumn{7}{|c|}{ E } \\
\hline & & \multirow{2}{*}{$\begin{array}{c}\mathrm{NB} \\
\mathrm{PB} / \mathrm{NB} / \mathrm{PS}\end{array}$} & \multirow{2}{*}{$\begin{array}{c}\mathrm{NM} \\
\mathrm{PB} / \mathrm{NB} / \mathrm{PS}\end{array}$} & \multirow{2}{*}{$\begin{array}{c}\text { NS } \\
\text { PM/NB/O }\end{array}$} & \multirow{2}{*}{$\begin{array}{c}0 \\
\mathrm{PM} / \mathrm{NM} / \mathrm{O}\end{array}$} & \multirow{2}{*}{$\begin{array}{c}\mathrm{PS} \\
\mathrm{PS} / \mathrm{NM} / 0\end{array}$} & \multirow{2}{*}{$\begin{array}{c}\mathrm{PM} \\
\mathrm{PS} / \mathrm{O} / \mathrm{PB}\end{array}$} & \multirow{2}{*}{$\begin{array}{c}\mathrm{PB} \\
\text { O/O/PB }\end{array}$} \\
\hline \multirow{7}{*}{$\mathrm{EC}$} & NB & & & & & & & \\
\hline & NM & $\mathrm{PB} / \mathrm{NB} / \mathrm{NS}$ & $\mathrm{PM} / \mathrm{NM} / \mathrm{NS}$ & $\mathrm{PM} / \mathrm{NM} / \mathrm{NS}$ & $\mathrm{PM} / \mathrm{NM} / \mathrm{NS}$ & $\mathrm{PS} / \mathrm{NS} / 0$ & O/O/NS & O/0/PM \\
\hline & NS & $\mathrm{PM} / \mathrm{NM} / \mathrm{NB}$ & $\mathrm{PM} / \mathrm{NM} / \mathrm{NB}$ & $\mathrm{PM} / \mathrm{NS} / \mathrm{NM}$ & PS/NS/NS & $0 / 0 / 0$ & NS/PS/PS & NM/PS/PM \\
\hline & 0 & $\mathrm{PM} / \mathrm{NM} / \mathrm{NB}$ & PS/NS/NB & $\mathrm{PM} / \mathrm{NS} / \mathrm{NM}$ & O/O/NS & NS/PS/O & NM/PS/PS & NM/PM/PM \\
\hline & PS & $\mathrm{PS} / \mathrm{NS} / \mathrm{NB}$ & PS/NS/NB & $0 / 0 / \mathrm{NS}$ & NS/PS/NS & NS/PS/0 & NM/PM/PS & $\mathrm{NM} / \mathrm{PM} / \mathrm{PS}$ \\
\hline & PM & O/O/NM & $0 / 0 / \mathrm{NM}$ & NS/PS/NS & NM/PM/NS & $\mathrm{NM} / \mathrm{PM} / 0$ & $\mathrm{NM} / \mathrm{PB} / \mathrm{PS}$ & $\mathrm{NB} / \mathrm{PB} / \mathrm{PS}$ \\
\hline & PB & $0 / 0 / P S$ & $0 / 0 / \mathrm{PS}$ & NS/PS/O & $\mathrm{NM} / \mathrm{PM} / \mathrm{O}$ & $\mathrm{NM} / \mathrm{PB} / \mathrm{O}$ & $\mathrm{NB} / \mathrm{PB} / \mathrm{PB}$ & $\mathrm{NB} / \mathrm{PB} / \mathrm{PB}$ \\
\hline
\end{tabular}

3) Fuzzy control rule tables

The fuzzy adaptive controller couldn't get a perfect control performance unless there's a complete fuzzy control rules, so choosing suitable fuzzy control rules is a key point. This paper selects Table I as the fuzzy control rules after study the functions of $K_{P}, K_{I}$ and $K_{D}$.

\section{NETWORKED CONTROL SYSTEM BASED ON FUZZY ADAPTIVE PID CONTROLLER}

The simulation model of fuzzy adaptive PID control system based on TrueTime is consisted of a network module and a number of computer modules, where computer modules are used as nodes of network. The simulation model is shown in Fig. 3, which is designed according Fig. 1 and TrueTime toolbox.

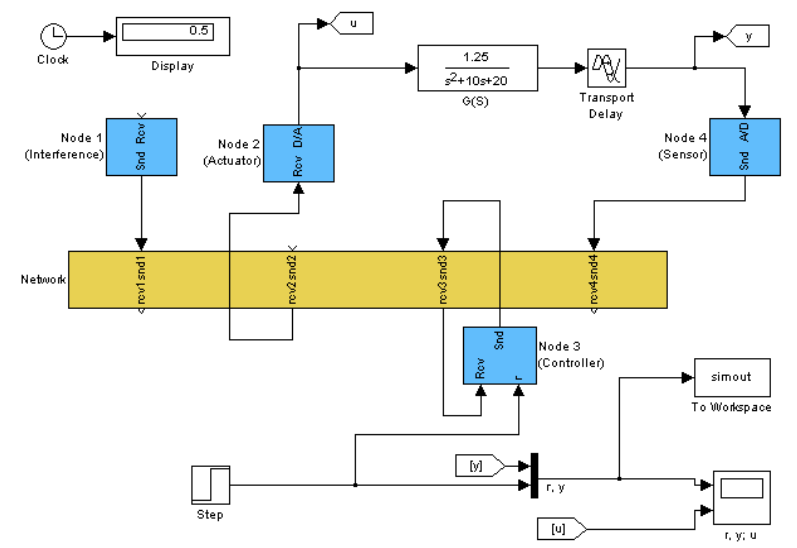

Fig. 3. The simulation model of fuzzy adaptive PID networked control system based on TrueTime.

The system includes four computer nodes, and each of them is represented by TrueTime kernel module. A sensor node (Node 4) is periodic sampling from the controlled object, and through the network module, the samples will be sent to the controller node (Node 3). The task of controller node is calculating the control signals by fuzzy adaptive PID control algorithm and sending the results to actuator node (Node 2), and the actuator node executes the control signals later. The system also contains an interference node (Node 1), which is used to produce a random interference signal to hinder the network transmission, and to simulate loads in the network, which send and receive data periodically, occupy the network bandwidth and make the network node involved in control have transmission delay. Prescribe that the signals produced by interference node has the highest priority, and the reference signal uses the square wave signal triggered at zero. The sensor node is set to clock driven mode (sampling period to drive the device to work), and the controller node and actuator node are set to event-driven mode (specific data reaching or sending over to drive devices to work).

The TrueTime network module receives and sends data in accordance with the network module selected, which can provide six kinds of modes and network parameters, such as the number of network nodes, transmission rate and medium access control protocol (MAC) and so on, where MAC includes CSMA/CD (Ethernet), CSMA/AMP, Round Robin, FDMA, TDMA, and Switched Ethernet, etc. The network communication mode can be changed through setting the Network type in the TrueTime Network module.

For the date packet sent in the networked control system is usually short and the frequency of sending and receiving date is always high, the phenomenon of information collision and retransmission is caused inevitably for the limited broadband, which leads the delay of information transmission. Meanwhile, a larger overshoot and longer control period, which affect the control quality seriously during production and bring down the system stability greatly, is caused by the stochastic change of network delay and the influence of the time delay factors, such as network protocol and network load, etc. The delay time is changed by Transport Delay in the simulation model of networked control system based on TrueTime. 


\section{Simulation STUdies}

This paper takes the typical second-order inertia plant in the process of industrial production as an example, whose transfer function is shown as belowing:

$$
G(s)=\frac{1.25}{s^{2}+10 s+20}
$$

During the simulation study, the sampling period is $0.01 \mathrm{~s}$, the network communication mode is CSMA/CD (Ethernet), and the transmission rate is $100 \mathrm{Kbit} / \mathrm{s}$. There are different simulations when the parameters of controlled object have been changed so as to verify the robustness and adaptability. In Fig. 4, curve (1) shows the result when input is a unit step signal, curve (2) is the response of unchanged parameters, curve (3) is the response curve when the controlled object is

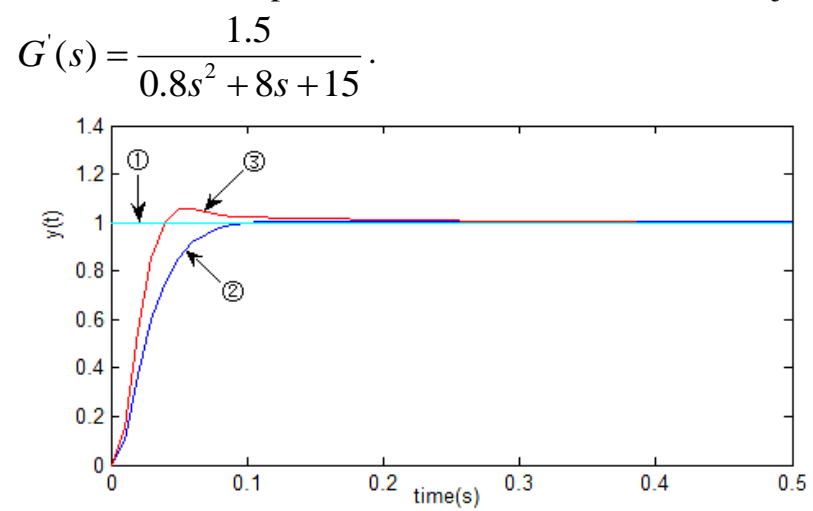

Fig. 4. The curves of simulation results comparison.

Fig. 5 shows the simulation results after having changed the network transmission rate. Curve (1) shows the result when input is a unit step signal, curve (2) shows the result when transmission rate is $30 \mathrm{Kbit} / \mathrm{s}$, curve (3) shows the result when transmission rate is $20 \mathrm{Kbit} / \mathrm{s}$, curve (4) shows the result when transmission rate is $15 \mathrm{Kbit} / \mathrm{s}$. Through these simulations, it can be improved that the overshoot is bigger and the oscillation is increasing when the transmission rate is reduced. The networked control system will be instability when the transmission rate is decreased a certain value.

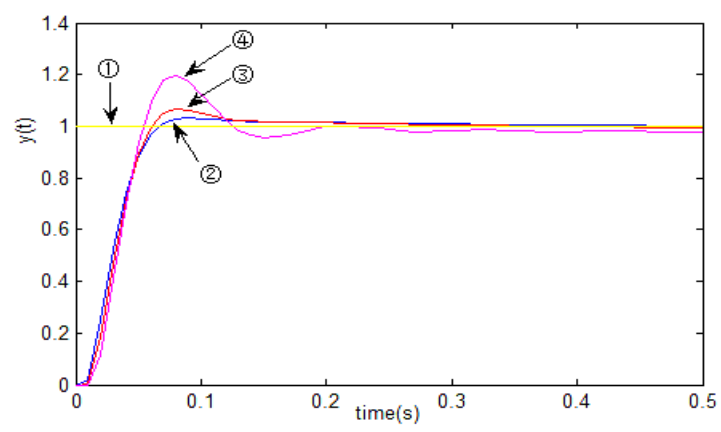

Fig. 5. The curves of results comparison after changing transmission rate.

Fig. 6 shows the simulation results after increasing a time delay. Curve (1) shows the result when input is a unit step signal, curve (2) shows the result when time delay is $0.005 \mathrm{~s}$, curve (3) shows the result when time delay is $0.01 \mathrm{~s}$, curve (4) shows the result when time delay is $0.015 \mathrm{~s}$. Through these simulations, it can be seen that the control system can keep stability and sill get a good control effect after increasing a certain time delay. However, the control effect will be worse with a time delay and the system may be instability if the time delay is too big.

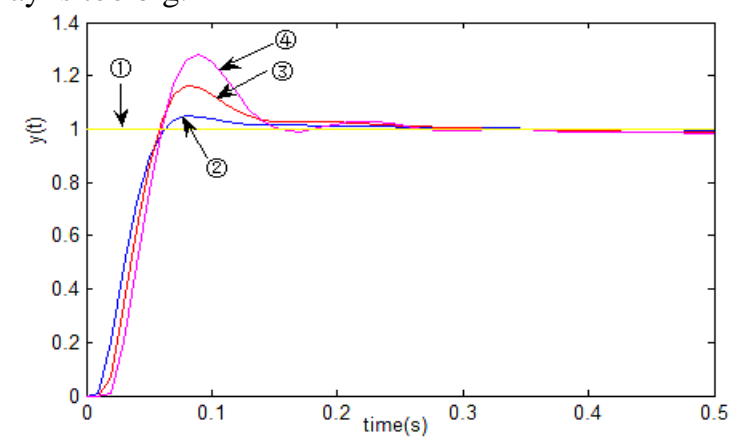

Fig. 6. The curves of results comparison after increasing time delay.

\section{CONCLUSIONS}

Networked control system has been a hotspot in the research fields of control theory and control engineering application at home and abroad. It has the advantages of fewer cables, easy to extend, high flexibility, resource sharing and remote controlling, etc. As the control object includes complicated control network and physical object, the controller of networked control system can adopt fuzzy control method. In this paper, fuzzy adaptive PID networked control system based on TrueTime is designed by combining the fuzzy control rules and PID controller. Take the typical second-order inertia plant in the process of industrial production as an example, and through the simulation study of changing parameters and transmission rates, increasing network delay, the feasibility and effectiveness of the control scheme had been proved.

\section{REFERENCES}

[1] X. D. Dang and Q. L. Zhang, "Stability and PID control for networked control system," in Proc. the $8^{\text {th }}$ World Congress on Intelligent Control and Automation, July 6-9 2010, Jinan, China, pp. 921-926.

[2] Y. Yin, S. F. Li, and J. H. Wang, "Research on Adaptive Fuzzy PID Control Method For Networked Control Systems," Journal of Wuhan University of Technology, 2010, vol. 32, no. 6, pp.144-148.

[3] B. Chen, C.M. Meng, and B. Ge et al., "Simulation Research on Networked Control System Based on Truetime," Control Engineering of China, vol. 15, no. 5, pp. 501-503, 2008.

[4] J. M. Sun, W. Deng, and L. P. Li et al., "Matlab based simulation platform for networked control system," Process Automation Instrumentation, 2010, vol. 31, no. 3, pp.19-22.

[5] D. G. Peng, H. Zhang, and J. J. Lin et al., "Research of Networked Control System Based on Fuzzy Immune PID Controller," Lecture Notes in Artificial Intelligence 7003, Part II, 2011, pp.141-148.

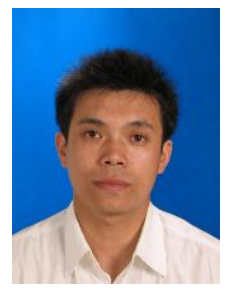

Daogang Peng received the BS degree in Control Theory and Control Engineering from North China Electric Power University, China in 2004, and Ph.D. degree in System Engineering from Tongji University in 2009. He is currently a professor in the School of Automation Engineering at Shanghai University of Electric Power and His main research interests include networked control system, intelligent control, fault diagnosis, embedded system, industrial Ethernet, etc.

Hao Zhang received the BS degree from Xi'an Jiaotong University, China in 1987, and Ph.D. degree from Shanghai Jiaotong University in 1990. He is currently a professor and vice president at Shanghai University of Electric Power. His main research interests include electrical power system automation, industrial Ethernet, systems engineering, etc. 